\title{
ZA Special Filter to Eliminate Salt and Pepper Noise from Gray and Color Images
}

\author{
Saleh A. Khawatreh, PhD \\ Al-Ahliyya Amman University \\ Faculty of engineering technology, \\ Jordan, Amman \\ Yousif Eltous \\ Professor \\ Albalqa Applied University \\ Faculty of Engineering Technology, Jordan, \\ Amman
}

\author{
Ziad Alqadi \\ Professor \\ Albalqa Applied University \\ Faculty of Engineering Technology, Jordan, \\ Amman
}

\begin{abstract}
Color images are the most important and most common digital data circulating between people and institutions due to their use in various computer applications, which requires removing noise from them to become clear and comfortable to the eye and to facilitate the process of processing them. In this paper research we will introduce a special ZA filter, which can efficiently used to eliminate salt and pepper noise from both gray and color images. The proposed filter will be tested using various images with various noise ratios; the PSNR and MSE values between the clean and de-noised will be calculated. The obtained experimental results will be compared median and average filters results in order to raise some judgments.
\end{abstract}

\section{Keywords}

RGB color image, gray image, median filter, average filter, salt and pepper noise, noise ratio, PSNR, MSE.

\section{INTRODUCTION}

Digital images [1], [2], whether gray [3], [4] or color [5], [6], have recently spread and have been used in many vital computer applications [7], [8], [9], such as the person's face recognition applications [10] or fingerprint [11], [12], [13] , to identify the person's identity. The images are also used in many security and protection systems [14], [15] and in medical applications and many other applications.

The clarity of the image and its freedom from noise is considered of great importance and the removal of noise, whether from the gray image or the color image, is one of the primary and primary processes for processing the digital image.

Noise reduces image quality resulting in loss of information and unsatisfactory visual effects. Salt and pepper noise is one of the most popular signals that affect image quality. In RGB color image, salt and pepper noise change how often colors overlap depending on the noise ratio. Several methods have been suggested to eliminate salt and pepper noise from the color image with minimal information loss. In this paper we will investigate the effects of adding salt and pepper noise to RGB color image, experimental noise ratio will be calculated and color combination will be calculated with the maximum and minimum presence in RGB color image. Additionally, this paper has proposed a methodology to eliminate salt and pepper noise for color images using an intermediate filter that provides image reconstruction in order to accept the result with minimal information loss. The proposed methodology will be applied and tested and experimental results will be analysed using the calculated values for MSE and PSNR [16], [17].

Salt-and-pepper noise is a form of noise sometimes seen on images. ... This noise can be caused by sharp and sudden disturbances in the image signal. It presents itself as sparsely occurring white and black pixels. An effective noise reduction method for this type of noise is a median filter or a morphological filter [18].

The negative effects of salt and pepper noise depend on the noise ratio, which the percent of effected pixel by the noise, the more noise ratio the more degradation on the image, figure 1 shows an image was affected by the noise with noise ratio equal 0.25 .

$4 \times 4$ original image :
$\left[\begin{array}{cccc}100 & 100 & 100 & 100 \\ 100 & 100 & 100 & 100 \\ 100 & 100 & 100 & 100 \\ 100 & 100 & 100 & 100\end{array}\right]$
image by impulse noise : $\left[\begin{array}{cccc}100 & 255 & 100 & 100 \\ 100 & 255 & 100 & 100 \\ 255 & 100 & 100 & 0 \\ 100 & 100 & 100 & 100\end{array}\right]$

Figure 1: Salt and pepper noise with ratio $=0.25$

To measure the quality of any method of salt and pepper removal we have to calculate the peak-to-signal-noise-ratio (PSNR) between the clear image and the de-noised one, the higher the PSNR value, the more efficient the method, or to calculate the mean square error (MSE) between the clean image and the de-noised one, and here the lower the MSE value, the more efficient the method.

In this paper research we will compare the results of the proposed method with most popularly used method: median filter and average filter methods, and we will show how the 
proposed ZA filter increases the value of PSNR and decreases the value of MSE.

\section{1- Proposed ZA filter}

The proposed ZA filter can be used to reduce salt and pepper noise from gray and color images, the salt and pepper noise point can be treated as isolated point or as local minimums and local maximums [20], and here we follow the steps of edge detection to detect the noise points and then remove them from the noisy image.

\section{- $\quad$ Applying ZA method for gray images}

To remove the noise from a gray image we have to execute the following steps as shown in figure 2 :

1) Get the noisy image.

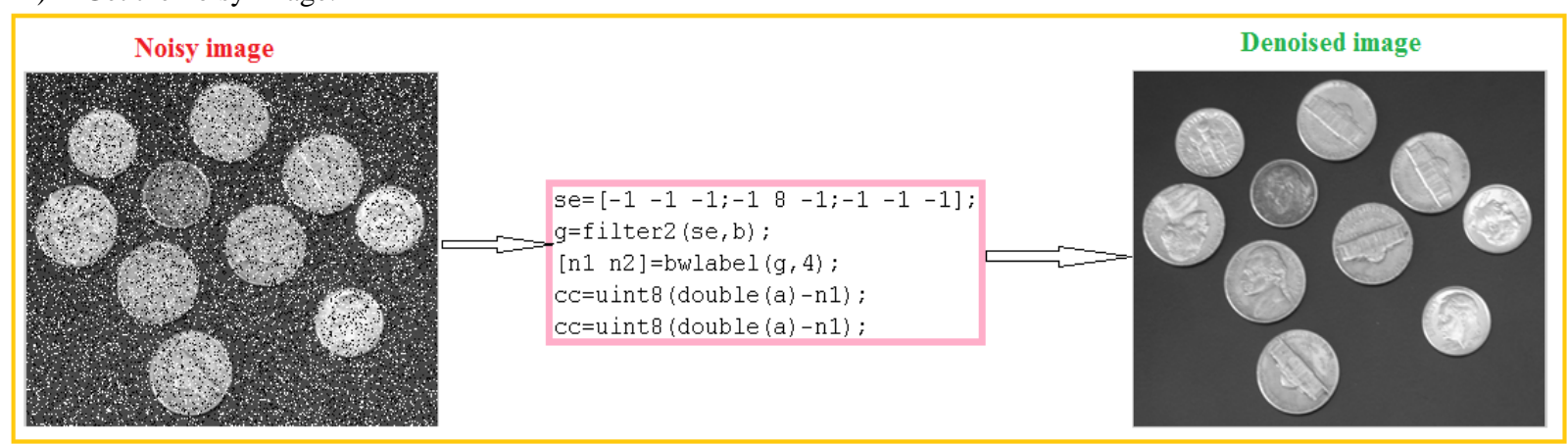

Figure 2: Applying ZA filter for gray images
Applying ZA method for color images

To remove the noise from a color image we have to execute the following steps as shown in figure 3 :

1) Get the noisy image.

2) Extract each color matrix

3) Select the following mask for isolated points detection and edge detection.

$\begin{array}{rrr}-1 & -1 & -1 \\ -1 & 8 & -1 \\ -1 & -1 & -1\end{array}$

2) Select the following mask for isolated points detection and edge detection.

$\begin{array}{rrr}-1 & -1 & -1 \\ -1 & 8 & -1 \\ -1 & -1 & -1\end{array}$

3) Apply correlation to get the image gradient.

4) Apply matlab function bwlabel to get the index matrix.

5) Subtract the index matrix from the noisy image matrix.
4) For each color matrix Apply correlation to get the image color gradient.

5) Apply matlab function bwlabel to get the index matrix for each color.

6) Subtract the index matrix from the noisy image matrix for each noisy color.

7) Merge the color matrix to get the de-noised color image

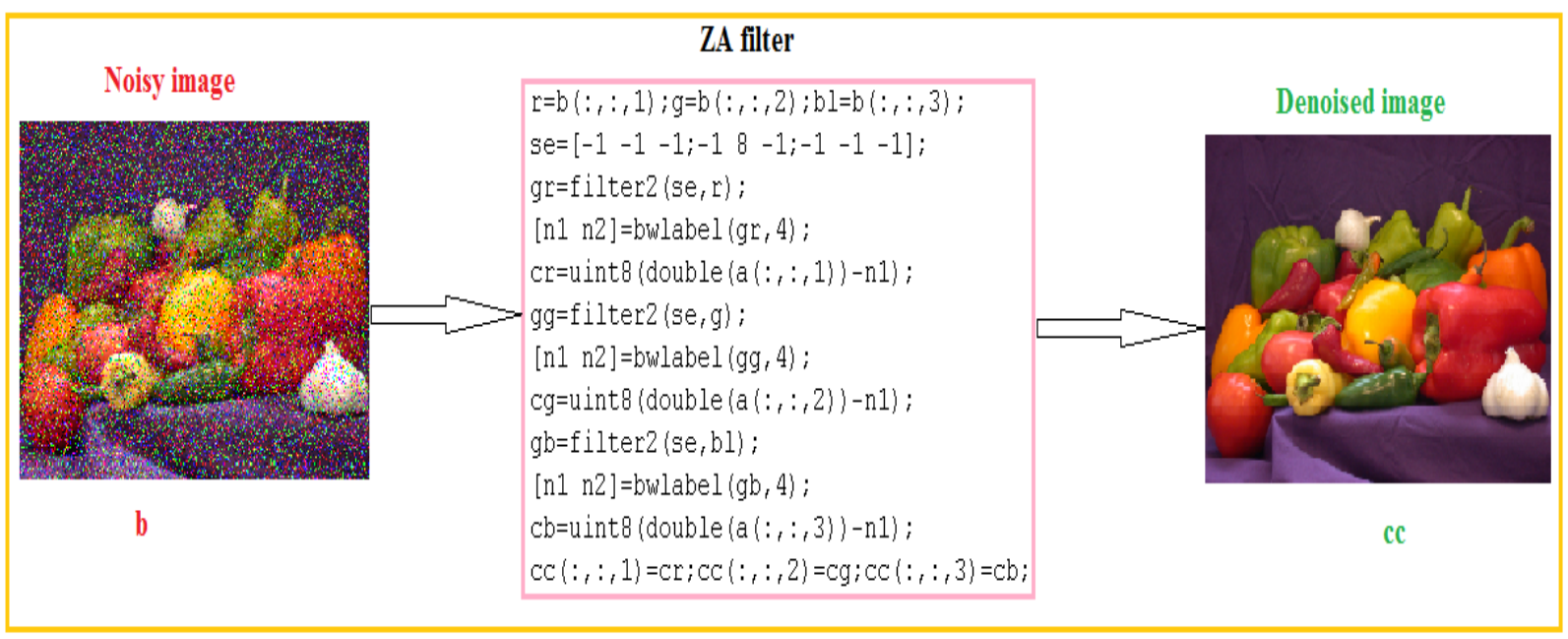

Figure 3: Applying ZA filter for color images 


\section{IMPLEMENTATION AND EXPERIMENTAL RESULTS}

The proposed ZA filter was implemented using gray images, the gray images were noised using salt and pepper noise, the noisy images then were filtered using ZA, median and average filters, figure 4 shows a gray image filtering example .
A pout.tif image was taken, and a salt and pepper noise was added to this image using different values of noise ratio, then the noisy image was treated using each of the three filters, table 1 shows the obtained experimental results.

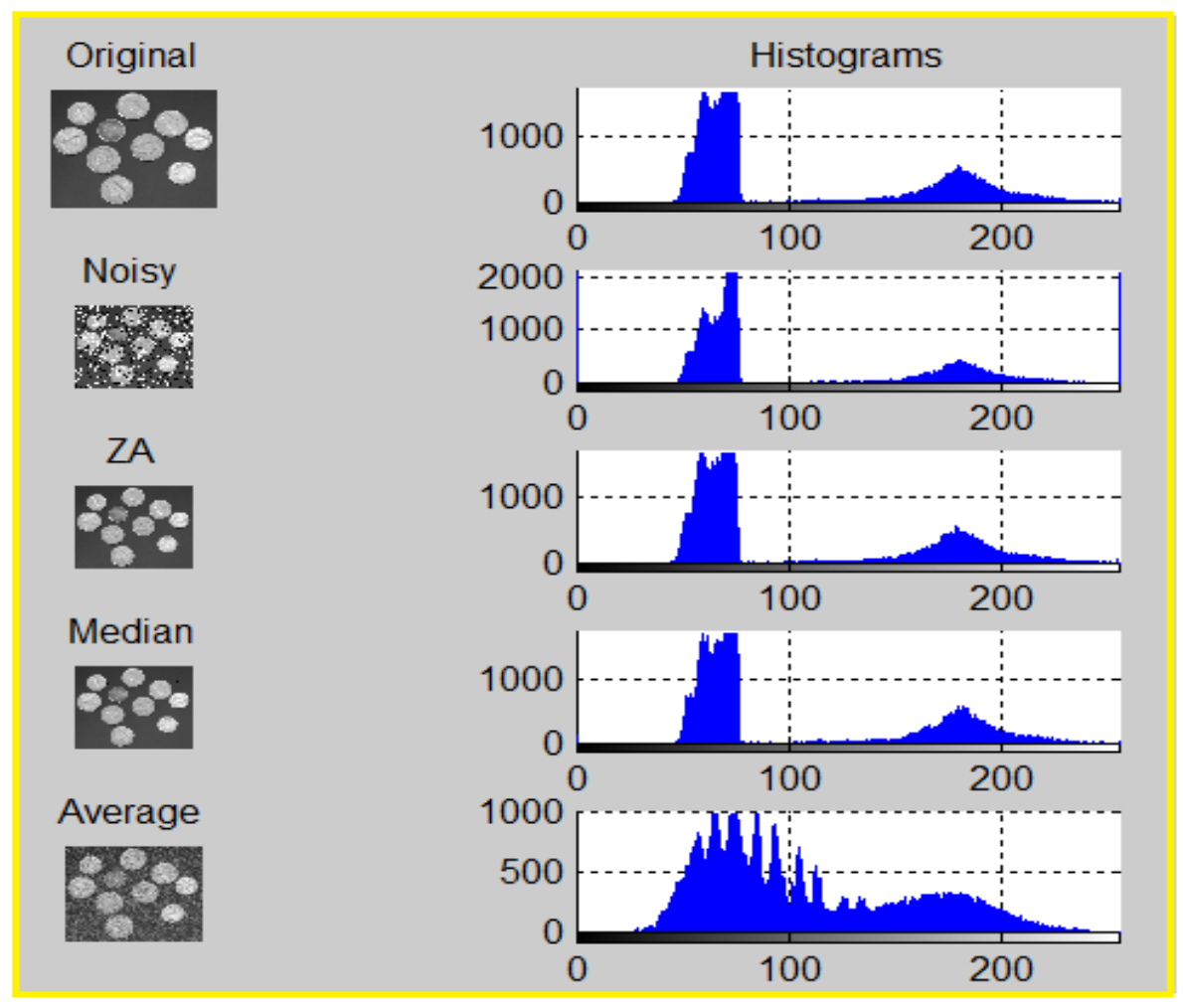

Figure 4: Gray image filtering example

Table 1: Gray image filtering results

\begin{tabular}{|c|c|c|c|c|c|c|}
\hline \multirow[t]{2}{*}{ Noise ratio } & \multicolumn{2}{|c|}{$\mathrm{ZA}$} & \multicolumn{2}{|c|}{ Median filter } & \multicolumn{2}{|c|}{ Average filter } \\
\hline & MSE & PSNR & MSE & PSNR & MSE & PSNR \\
\hline 0.002 & 0.9694 & 47.1397 & 0.9694 & 40.4978 & 4.4742 & 32.6573 \\
\hline 0.005 & 0.9704 & 47.1352 & 4.4884 & 40.4841 & 33.3645 & 31.7721 \\
\hline 0.01 & 0.9717 & 47.1295 & 4.6762 & 40.3060 & 42.6949 & 30.7012 \\
\hline 0.02 & 0.9743 & 47.1180 & 5.3462 & 39.7245 & 62.0268 & 29.1947 \\
\hline 0.03 & 0.9768 & 47.1067 & 4.9870 & 40.0265 & 80.3023 & 27.9577 \\
\hline 0.04 & 0.9784 & 47.0999 & 6.9872 & 38.5619 & 99.2213 & 27.0389 \\
\hline 0.05 & 0.9801 & 47.0922 & 6.3059 & 39.0075 & 118.8801 & 26.2926 \\
\hline 0.09 & 0.9867 & 47.0632 & 12.1901 & 37.2707 & 198.8300 & 24.0201 \\
\hline 0.1 & 0.9873 & 47.0605 & 12.7701 & 37.0688 & 219.5842 & 23.5890 \\
\hline 0.2 & 0.9948 & 47.0275 & 56.9959 & 30.5724 & 421.5584 & 21.4277 \\
\hline
\end{tabular}

From table 1 we can see that ZA filter provides the highest value for PSNR and the lowest values for MSE, thus ZA filter can be used to enhance the process of removing salt and pepper noise from a gray level. From table 1 we can see that ZA filter keeps PSNR high and MSE low even if the noise ratio is high as you see in figure 5 . 


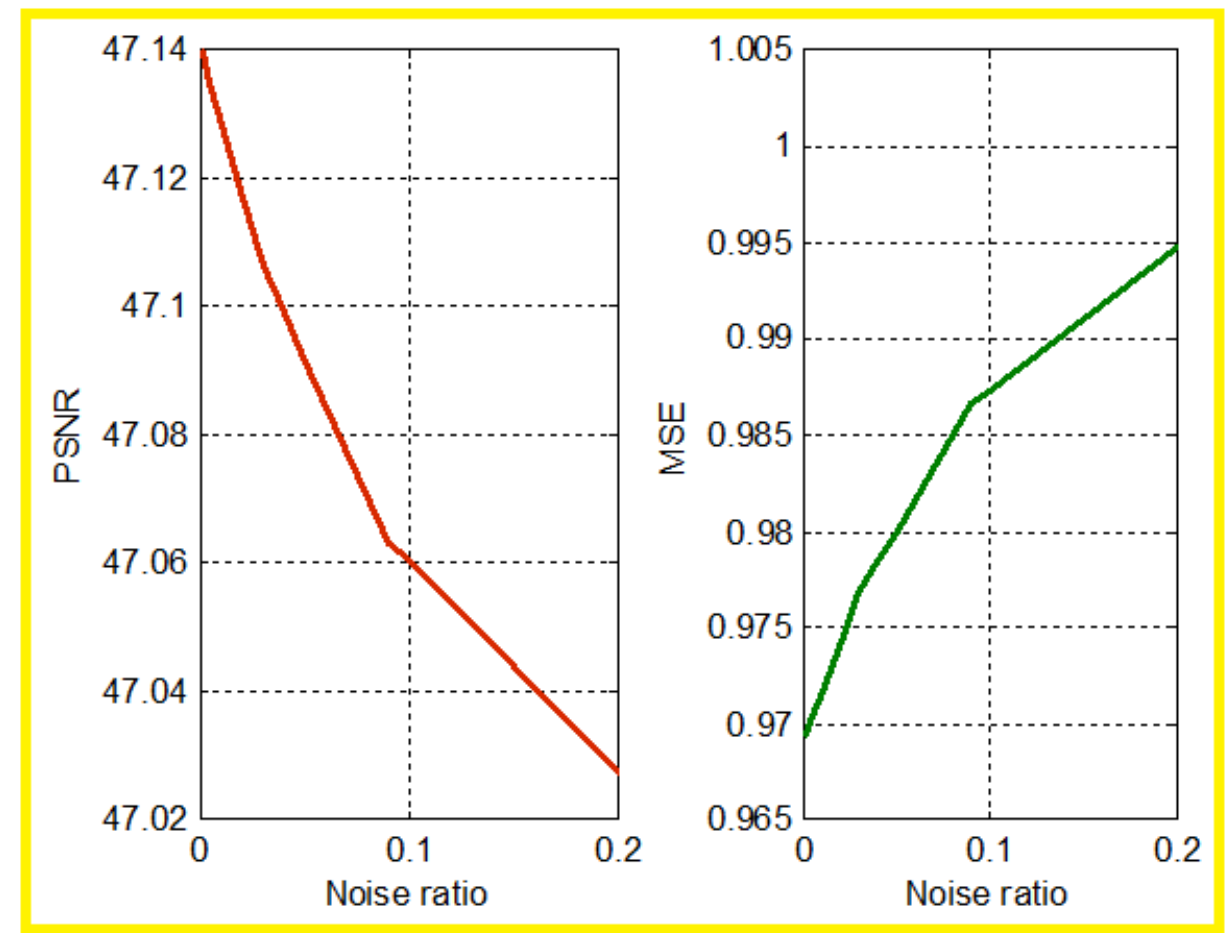

Figure 5: ZA filter features

The proposed ZA filter was also implemented using color images, the color images were noised using salt and pepper noise, the noisy images then were filtered using ZA, median and average filters, figure 5 shows a color image filtering example .
A peppers.png image was taken, and a salt and pepper noise was added to this image using different values of noise ratio, then the noisy image was treated using each of the three filters, table 2 shows the obtained experimental results.

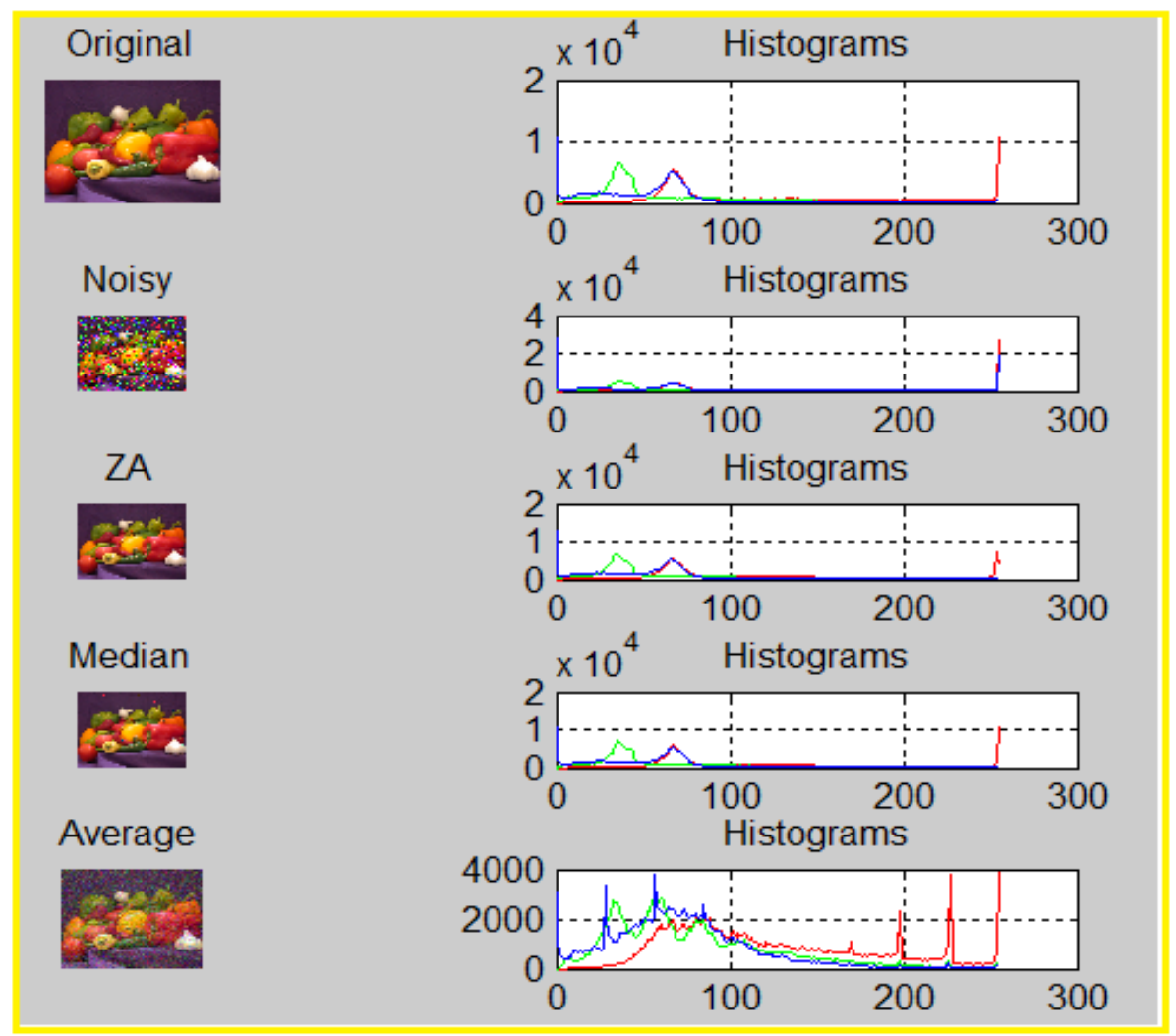

Figure 6: Color image filtering example 
Table 2: Color image filtering results

\begin{tabular}{|c|c|c|c|c|c|c|}
\hline \multirow[t]{2}{*}{ Noise ratio } & \multicolumn{2}{|c|}{$\mathrm{ZA}$} & \multicolumn{2}{|c|}{ Median filter } & \multicolumn{2}{|c|}{ Average filter } \\
\hline & MSE & PSNR & MSE & PSNR & MSE & PSNR \\
\hline 0.002 & 0.9503 & 111.3347 & 8.5810 & 89.3298 & 25.4913 & 89.3298 \\
\hline 0.005 & 0.9524 & 111.3127 & 8.6606 & 89.2374 & 32.7254 & 75.9438 \\
\hline 0.01 & 1.1646 & 109.3013 & 8.8385 & 89.0340 & 45.3483 & 72.6815 \\
\hline 0.02 & 1.6583 & 105.7675 & 9.4642 & 88.3501 & 72.3158 & 68.0148 \\
\hline 0.03 & 2.8777 & 100.2554 & 9.9144 & 87.8854 & 101.0541 & 64.6687 \\
\hline 0.04 & 4.1855 & 96.5090 & 10.5040 & 87.3077 & 127.8458 & 62.3170 \\
\hline 0.05 & 5.0805 & 94.5712 & 10.9936 & 86.8521 & 157.0595 & 60.2590 \\
\hline 0.09 & 7.9401 & 90.1060 & 14.8847 & 83.8219 & 286.3645 & 54.2526 \\
\hline 0.1 & 6.2330 & 92.5268 & 16.7230 & 82.6574 & 317.6584 & 53.2155 \\
\hline 0.2 & 0.9756 & 111.0724 & 68.8024 & 68.5129 & 718.2172 & 45.0576 \\
\hline 0.5 & 0.9803 & 111.0244 & 2251.1 & 33.6335 & 2556.6 & 32.3609 \\
\hline
\end{tabular}

ZA filter also was experimented using color images with various sizes by fixing the noise ratio to 0.5 , figure 7 shows the result of filtering a color image of big size, while table 3 shows the results of treating various images using the three filters, and here we can see that ZA filter provides the best values for PSNR and MSE.

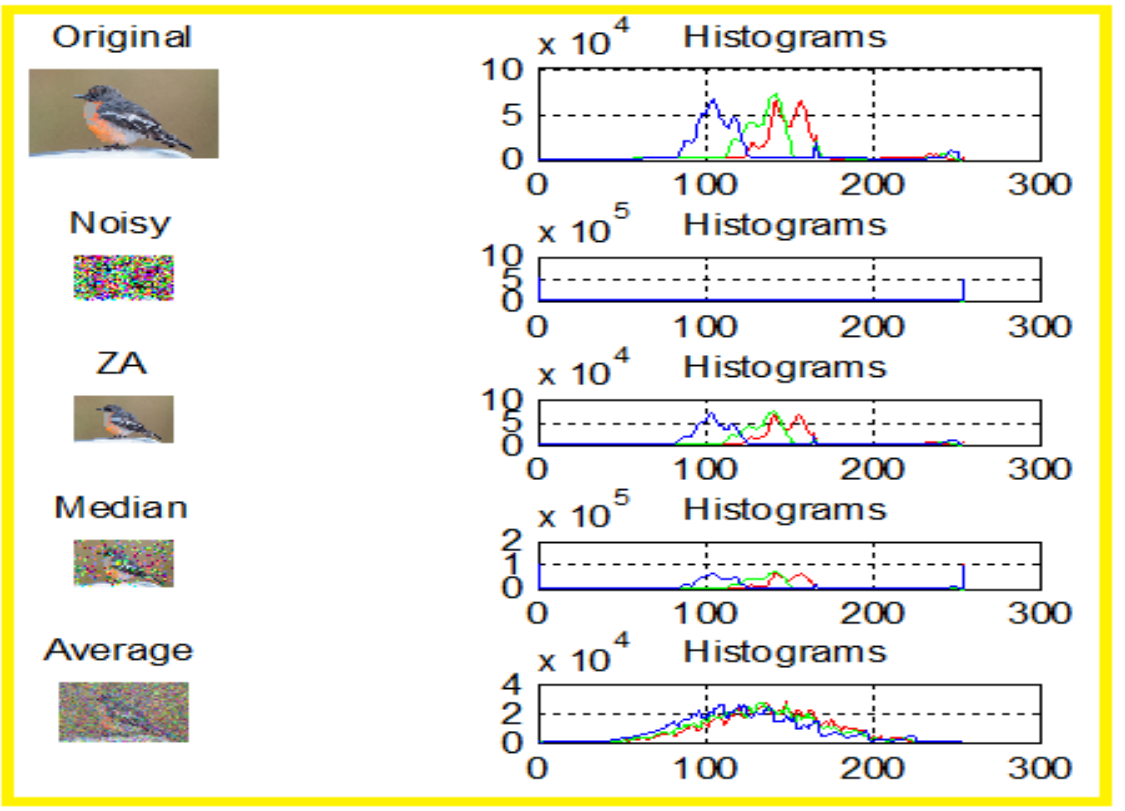

Figure 7: Filtering a big image

Table 3: Various color image filtering results

\begin{tabular}{|c|c|c|c|c|c|c|}
\hline \multirow[t]{2}{*}{ Image size(byte) } & \multicolumn{2}{|c|}{$\mathrm{ZA}$} & \multicolumn{2}{|c|}{ Median filter } & \multicolumn{2}{|c|}{ Average filter } \\
\hline & MSE & PSNR & MSE & PSNR & MSE & PSNR \\
\hline 151875 & 0.9503 & 110.8376 & 2145.9 & 34.1121 & 1861.4 & 35.5347 \\
\hline 150738 & 0.9992 & 108.8561 & 1896.2 & 35.3490 & 1381.1 & 37.8004 \\
\hline 151050 & 0.9849 & 110.9778 & 2638.0 & 32.0476 & 3026.9 & 30.6725 \\
\hline 151008 & 0.9901 & 110.9252 & 3059.2 & 30.5662 & 2872.8 & 31.1948 \\
\hline 151515 & 0.9870 & 110.9561 & 2380.1 & 33.0761 & 2006.0 & 34.7862 \\
\hline 150849 & 0.9714 & 111.1151 & 3613.0 & 28.9024 & 3569.0 & 29.0249 \\
\hline
\end{tabular}




\begin{tabular}{|l|l|l|l|l|l|l|}
\hline 5140800 & 0.9858 & 110.9683 & 2155.8 & 34.0660 & 2136.0 & 34.1584 \\
\hline 6119256 & 0.9992 & 110.8336 & 1809.3 & 35.8185 & 1376.0 & 38.5560 \\
\hline 2500608 & 0.9535 & 111.3018 & 3632.1 & 28.8496 & 3450.9 & 29.3613 \\
\hline \multicolumn{7}{|c|}{ Average } \\
\hline 1629700
\end{tabular}

From table 3 we can see that ZA filter adds and enhancement to the process of removing salt and pepper noise, this enhancement can be calculated by dividing PSNR for ZA filter by PSNR of other filter, the enhancement is greater than one as shown in table 4 , and from here we can strongly recommend ZA filter to remove salt and pepper noise from both gray image, regardless the image size and the noise ratio.

Table 4: ZA filter enhancement

\begin{tabular}{|c|c|c|c|}
\hline Method & ZA filter & Median filter & Average filter \\
\hline ZA filter & 1 & 3.4044 & 3.3105 \\
\hline Median filter & 0.2937 & 1 & 0.9724 \\
\hline Average filter & 0.3021 & 1.0284 & 1 \\
\hline
\end{tabular}

\section{CONCLUSION}

A ZA filter of salt and pepper removing was proposed, implemented and tested. The experimental results showed that the proposed filter can be used for both gray and color images. The proposed filter was compared with median and average filters, and it was shown that ZA filter provides the best values for PSNR and MSE.

The proposed ZA filter add an enhancement to the process of filtering and it can by highly recommended for removing salt and pepper noise from gray and color images.

\section{REFERENCES}

[1] Majed O Al-Dwairi, Ziad A Alqadi, Amjad A Abujazar, Rushdi Abu Zneit, Optimized true-color image processing, World Applied Sciences Journal, vol. 8, issue 10, pp. 1175-1182, 2010.

[2] Jamil Al Azzeh, Hussein Alhatamleh, Ziad A Alqadi, Mohammad Khalil Abuzalata, Creating a Color Map to be used to Convert a Gray Image to Color Image, International Journal of Computer Applications, vol. 153, issue 2, pp. 31-34, 2016.

[3] Akram A. Moustafa and 2Ziad A. Alqadi, Color Image Reconstruction Using A New R'G'I Model, Journal of Computer Science 5 (4): 250-254, 2009.

[4] Waheeb Abu Ulbeh, Akram Moustafa, Ziad A Alqadi, Gray image reconstruction, European Journal of Scientific Research, vol. 27, issue 2, pp.167-173, 2009.

[5] Ziad Alqadi, A Novel Methodology for Repairing a Torn Image, International Journal on Communications Antenna and Propagation, vol. 4, issue 1, pp. 366-372, 2011.

[6] Amjad Y Hindi, Majed O Dwairi, Ziad A AlQadi, Creating Human Speech Identifier using WPT, International Journal of Computer Science and Mobile Computing, vol. 9, issue 2, pp. 117-123, 2020.

[7] J Al-Azzeh M Abuzalata, Ziad Alqadi, Modified Inverse LSB Method for Highly Secure Message Hiding, International Journal of Computer Science and Mobile Computing, vol. 8, issue 2, pp. 93-103, 2019.
[8] Prof. Mohammed Abu Zalata Dr. Ghazi. M. Qaryouti, Dr.Saleh Khawatreh, Prof. Ziad A.A. Alqadi, Optimal Color Image Recognition System (OCIRS), International Journal of Advanced Computer Science and Technology, vol. 7, issue 1, pp. 91-99, 2017.

[9] Aws AlQaisi, Mokhled AlTarawneh, Ziad A. Alqadi, Ahmad A. Sharadqah, Analysis of Color Image Features Extraction using Texture Methods, TELKOMNIKA, Vol.17, No.3, pp. 1220-1225, 2019.

[10] Prof. Ziad Alqadi Dr. Mohammad S. Khrisat, Prof. Yousif Eltous, Dr. Saleh A. Khawatreh, Dr. Majed Omar Dwairi, Building Face Recognition System (FRS), International Journal of Computer Science and Mobile Computing, vol. 9, issue 6, pp. 15-24, 2020.

[11] Dr. Amjad Hindi, Dr. Majed Omar Dwairi, Prof. Ziad Alqadi, Analysis of Procedures used to build an Optimal Fingerprint Recognition System, International Journal of Computer Science and Mobile Computing, vol. 9, issue 2, pp. $21-37,2020$.

[12] Ziad A. AlQadi Amjad Y. Hindi, Majed O. Dwairi, Analysis of Fingerprint Minutiae to form Fingerprint Identifier, IJCSMC, vol. 9, issue 2, pp. 38-48, 2020.

[13] Ziad Abdel AlQadi, Yousf Eltous, Mohammad Abuzalata, Ghazi M Qaryouti, Detecting and counting minutiae in human fingerprint, Open Science journal, vol. 5, issue 1, pp. 1-5, 2020.

[14] Musbah J. Aqel, Ziad A. Alqadi, Ibraheim M. El Emary, Analysis of Stream Cipher Security Algorithm, Journal of Information and Computing Science Vol. 2, No. 4, 2007, pp. 288-298.

[15] Belal Zahran Rashad J Rasras, Ziad Alqadi, Mutaz Rasmi Abu Sara, Developing new multilevel security algorithm for data encryption-decryption (MLS_ED), International Journal of Advanced Trends in Computer Science and Engineering, vol. 8, issue 6, pp. 3228-3235. 2019.

[16] Ziad Alqadi, Bilal Zahran, Jihad Nader, Estimation and Tuning of FIR Lowpass Digital Filter Parameters, International Journal of Advanced Research in Computer 
Science and Software Engineering, vol. 7, issue 2, pp. 18-23, 2017.

[17] Haitham Alasha'ary, Abdullah Al-Hasanat, Khaled Matrouk, Ziad Al-Qadi, Hasan Al-Shalabi, A Novel Digital Filter for Enhancing Dark Gray Images, European Journal of Scientific Research, vol. 122, issue 2, pp. 99106, 2014.

[18] Jamil Azzeh, Bilal Zahran, Ziad Alqadi, Salt and Pepper Noise: Effects and Removal, JOIV: International Journal on Informatics Visualization, vol. 2, issue 4, pp. 252-256, 2018.
[19] Jamil Al-azzeh Ahmad Sharadqh, Belal Ayyoub, Ziad Alqadi, Experimental investigation of method used to remove salt and pepper noise from digital color image, International Journal of Research in Advanced Engineering and Technology, vol. 5, issue 1, pp. 23-31, 2019.

[20] Akram A Moustafa, Ziad A Alqadi, A Practical Approach of Selecting the Edge Detector Parameters to Achieve a Good Edge Map of the Gray Image, Journal of Computer Science 5 (5): 355-362, 2009. 